\title{
A high productivity and speedy synthesis process for copper nanowires via an ethylenediamine-mediated method
}

\author{
Thanh-Hung Duong ${ }^{1} \cdot$ Hyun-Chul Kim ${ }^{1}$
}

Received: 23 September 2016/ Accepted: 20 February 2017/Published online: 9 March 2017

(c) The Author(s) 2017. This article is published with open access at Springerlink.com

\begin{abstract}
Generally, there are two well-known synthetic approaches for copper nanowires ( $\mathrm{Cu} \mathrm{NWs}$ ): ethylenediamine (EDA)-mediated synthesis and alkylamine-mediated synthesis. The alkylamine-mediated synthesis produces very high ratio nanowires but requires a special environment and long reaction times, while the EDA-mediated synthesis can be carried out under normal conditions and requires from $30 \mathrm{~min}$ to $1 \mathrm{~h}$. However, the $\mathrm{Cu}$ NWs produced by this method have an average aspect ratio lower than 500 and are produced in low yield. In this paper, we present a modified EDA-mediated synthesis to improve the yield and reduce the synthesis time. In previous reported EDA-mediated synthesis, sodium hydroxide $(\mathrm{NaOH})$ was used as a $\mathrm{pH}$ adjusting element and the reaction was performed at high temperature. By replacing $\mathrm{NaOH}$ with potassium hydroxide $(\mathrm{KOH})$ and cooling down the temperature of reaction to room temperature, the synthesis time was reduced to $15 \mathrm{~min}$ as well as the productivity of high aspect ratio $\mathrm{Cu}$ NWs was increased notably to $80 \%$. Furthermore, the transparent electrodes which were fabricated based on the as-synthesized Cu NWs, exhibited high performance, such as $23.5 \Omega / \mathrm{sq}$ of sheet resistance and $81 \%$ of transmittance at $\lambda=550 \mathrm{~nm}$.
\end{abstract}

Keywords Nanomaterials synthesis · Productivity · Copper nanowires $\cdot$ Ethylenediamine $\cdot$ Transparent electrode

Hyun-Chul Kim

mechkhc@inje.ac.kr

1 Department of Mechanical and Automotive Engineering, High Safety Vehicle Core Technology Research Center, Inje University, Gimhae-si, South Korea

\section{Introduction}

Currently, indium tin oxide (ITO) is the dominant transparent conductor material in various optoelectronic devices such as organic light emitting diodes (OLEDs), flexible solar cells, and touch-screens. The high transmittance ( $>90 \%$ ) of ITO at low sheet resistances (10 $\Omega /$ sq on glass) is the primary reason for its popularity. However, ITO has several undesirable attributes [1]. ITO is a ceramic, and is, therefore, brittle and prone to cracking. The abundance of indium in the earth's crust is low (0.05 ppm), and its cost is correspondingly high, reaching approximately US $\$ 700 / \mathrm{kg}$ in 2014 [2]. To solve these problems, various types of transparent electrodes based on nanoscale materials have been developed over the past twelve years [3, 4]. Recently, as an alternative to ITO, copper nanowires (CuNWs) have been studied extensively for use in fabricating transparent conductors.

Copper has extremely low resistivity, $1.7 \times 10^{-8} \Omega \mathrm{m}$ at $293.15 \mathrm{~K}$, higher than only silver and carbon graphene. Its price was US\$5.22/kg in 2015, 100 times less expensive than silver and indium [5]. Many reported studies show remarkable performance of CuNWs thin films. Over the past decade, there are two general synthesis methods for CuNWs: ethylenediamine (EDA)-mediated synthesis and alkylamine-mediated synthesis. The alkylamine-mediated synthesis is usually carried out under neutral or moderately basic conditions at a high temperature, under a high pressure; this synthesis requires at least $10-12 \mathrm{~h} \mathrm{[6-9].} \mathrm{The}$ CuNWs produced by this method have diameters of $30-100 \mathrm{~nm}$ and lengths up to several millimeters. On the other hand, EDA-mediated syntheses are several-fold faster, requiring less than an hour, and can be performed under atmospheric pressure [10-14]. However, the aspect ratios of CuNWs produced by this method are typically lower 
than those produced by alkylamine-based synthesis. In 2014, Ye et al. developed a rapid method that required only 30 min and afforded high aspect ratio CuNWs with an average L/D of 1860 [10]. While the yield of CuNWs produced by the EDA-mediated method is low, about $12 \%$, Ye et al. developed a novel two-pots method to improve the production of CuNWs by using cuprous oxide seeds, resulting in a yield of 55\% [11]. However, this method is complicated and requires a longer time.

In this paper, we present an edited EDA-mediated synthesis that is simple, rapid, and gives high yields of high aspect ratio $\mathrm{Cu}$ NWs. Generally, EDA-mediated method uses sodium hydroxide as a $\mathrm{pH}$ adjusting element $(\mathrm{NaOH})$ and keeps the reaction at high temperature from 60 to $80{ }^{\circ} \mathrm{C}$. Herein, we used potassium hydroxide $(\mathrm{KOH})$ as base source and maintained reaction temperature at room temperature. Moreover, the as-synthesized $\mathrm{Cu}$ NWs was coated onto soda lime glasses to make transparent electrodes.

\section{Experimental}

\section{Synthetic procedures}

To synthesize $\mathrm{Cu}$ NWs, $\mathrm{KOH}(15 \mathrm{M}, 20 \mathrm{~mL}), \mathrm{CuCl}_{2}$ $(0.1 \mathrm{M}, 1 \mathrm{~mL})$, and EDA $(2.25 \mathrm{mmol})$, were placed in a $50 \mathrm{~mL}$ flask and stirred at $650 \mathrm{rpm}$ for $2 \mathrm{~min}$ while retaining the mixture temperature at $60{ }^{\circ} \mathrm{C} . \mathrm{N}_{2} \mathrm{H}_{4}(35 \mathrm{wt} \%$, $15 \mu \mathrm{L}$ ) was then gently added dropwise, and the mixture was stirred for an additional $1 \mathrm{~min}$. Then the mixture was left to cool down at room temperature $\left(\mathrm{RT}, 25^{\circ} \mathrm{C}\right)$ until a reddish cake was formed. Next, the cake was transferred to a $50 \mathrm{~mL}$ tube and washed with $10 \mathrm{~mL}$ aqueous solution consisting of $1 \mathrm{wt} \%$ polyvinylpryrrolidone (PVP, MW $30,000)$ and $1 \mathrm{wt} \%$ diethylhydroxylamine (DEHA). The $\mathrm{Cu}$ NWs were washed with the above solution 3 times by centrifuge at $2000 \mathrm{rpm}, 5 \mathrm{~min}$ and stored in the same solution.

\section{Mass measuring}

Furthermore, due to the increase in the thickness of the $\mathrm{Cu}$ NWs cake in the preliminary experiments, a short experiment was carried out to measure the mass of the synthesized $\mathrm{Cu}$ NWs. To increase the measurement accuracy, all experiments were conducted with double the volume used in the previous experiments. After the cake was formed, it was washed 3 times with deionized (DI) water. $\mathrm{HCl}$ solution $(10 \mathrm{~mL}, 6 \%)$ was then added to remove unwanted organic impurities and copper (II) oxide. The mixture was centrifuged at $4000 \mathrm{rpm}$ for $10 \mathrm{~min}$ to remove residual $\mathrm{HCl}$, copper chloride, and unwanted chemicals. The mixture was washed once again with DI water. Following this, the mixture was filtered under vacuum to obtain pure $\mathrm{Cu}$ NWs on a polytetrafluoroethylene (PTFE) membrane with a pore size of $0.2 \mu \mathrm{m}$. The difference in the mass of the membrane before and after filtration indicated the mass of $\mathrm{Cu}$ NWs produced by this synthesis.

The yield of synthesis process was calculated by the following express:

Yield $=\frac{\text { Mass of as-synthesized CuNWs }}{\text { Mass of used } \mathrm{Cu}} \times 100 \%$.

\section{Copper nanowires electrodes preparation}

Generally, the $\mathrm{Cu}$ NWs electrodes preparation, which was carried out in this study, is similar with the one of Steward et al. [15].

First, nitrocellulose, also known as cellulose nitrate, was synthesized. $10 \mathrm{~mL}$ mixture of $\mathrm{H}_{2} \mathrm{SO}_{4} 98 \%$ and $\mathrm{HNO}_{3}$ $70 \%$ (ratio 7:3) was prepared in ice bath. Then $0.5 \mathrm{mg}$ cotton was added to this mixture. To make sure all the cotton was fully wet, the mixture was stirred hardly at $700 \mathrm{rpm}$ for $30 \mathrm{~min}$ at room temperature. After being fished out, the cotton was washed thoroughly in sodium bicarbonate solution 2 times and in DI water 3 times to neutralize the $\mathrm{pH}$ of cotton. Then the cotton was left in air for $6 \mathrm{~h}$ to dry completely. Next, to prepare the nitrocellulose-based ink, $0.24 \mathrm{mg}$ nitrocellulose was dissolved in $11.76 \mathrm{mg}$ acetone with hardly stirring for $15 \mathrm{~min}$. Then $12 \mathrm{~g}$ ethanol, $2 \mathrm{~g}$ ethyl acetate, $4 \mathrm{~g}$ pentyl acetate, $4 \mathrm{~g}$ isopropanol, and $6.8 \mathrm{~g}$ toluene were added, respectively. The nitrocellulose-based ink was formed by stirring this mixture for $10 \mathrm{~min}$.

Before mixing with the nitrocellulose-based ink, the stored $\mathrm{Cu}$ NWs sample needed to be purified from PVP and DEHA. This cleaning step was carried out by washing the sample 3 times with DI water and 2 times with ethanol. Then, $\mathrm{Cu}$ NWs were transferred to an aliquot $(1 \mathrm{~mL})$ and washed with nitrocellulose ink once. After pouring off the ink wash supernatant, $0.5 \mathrm{~mL}$ nitrocellulose-based ink was added to the $\mathrm{Cu}$ NWs to make the final coating solution. Next, $30 \mathrm{~mL}$ of this solution was pipetted in a small line across the top of the glass substrate $(7 \mathrm{~cm} \times 2.5 \mathrm{~cm})$. Right after that, a Meyer rod was rapidly and manually drawn down the substrate to equally disperse the copper nanowires coating solution.

Due to being covered by nitrocellulose-based ink, the $\mathrm{Cu}$ NWs film requires a post-treatment step to be conductive. First, the coated film was dried in air for $10 \mathrm{~min}$ at $80{ }^{\circ} \mathrm{C}$, then was left to cool down to room temperature. Next, it was plunged gently in glacial acetic acid for $2 \mathrm{~s}$, dried under air, and kept in oven at $80{ }^{\circ} \mathrm{C}$ for $45 \mathrm{~s}$. After four cycles of this procedure, the film was cooled down to 
room temperature and then dipped in glacial acetic acid and dried under air twice more without additional oven drying. Finally, the post-treated $\mathrm{Cu}$ NWs film was stored under $\mathrm{N}_{2}$ gas to avoid oxidation.

\section{Results and discussion}

\section{Effect of $\mathrm{KOH}$ and cooling to room temperature $\left(25^{\circ} \mathrm{C}\right)$}

First, it took only $15 \mathrm{~min}$ to form a reddish cake of $\mathrm{Cu}$ NWs by using our method. From the author's knowledge, no synthesis method of $\mathrm{Cu}$ NWs is quicker than ours. Normally, the alkylamine-mediated syntheses usually require from $6 \mathrm{~h}$ to several days [6-9]; while among ethylenediamine-mediated synthesis methods, the shortest time to form a $\mathrm{Cu}$ NWs cake is $30 \mathrm{~min}$ [10-14]. Until now, the mechanism underlying EDA-mediated synthesis of $\mathrm{Cu}$ NWs process has remained unclear; however, it is confirmed in previous studies that there are three vital elements necessary to form CuNWs. First, at least one $\mathrm{pH}$ adjusting species to form a first solution to make a suitable environment is required. Second, the reducing agent is used to reduce copper (II) ion into copper particle. Third, the capping agent which has skilled abilities to prevent copper atoms from freely growing, and help them to grow uniformly also plays key role in $\mathrm{Cu}$ NWs synthesis. A concentrated $\mathrm{NaOH}$ solution was commonly used to prevent the formation of copper hydroxide precipitates from copper ions [13]. However, once the concentration of $\mathrm{NaOH}$ reached $15 \mathrm{M}$, it became more difficult to achieve complete dissolution, and solid $\mathrm{NaOH}$ pieces remaining within the solution cover the copper atom, prevent them from contacting with the capping agent EDA, and finally caused the production of copper particles only [12]. On the other hand, $\mathrm{KOH}$ flakes easily dissolved in water to form a $15 \mathrm{M}$ solution; hence, it is more suitable for raising the $\mathrm{pH}$ of the solution and enhancing the reduction of copper by hydrazine. This may be the reason for the outstanding result observed when using $\mathrm{KOH}$ instead of $\mathrm{NaOH}$ : a significant reduction in reaction time.

Next, the as-synthesized Cu NWs were analyzed using a scanning electron microscope (SEM, Hitachi S-4800). As shown in Fig. 1 The average diameter and length of CuNWs were about $71.43 \mathrm{~nm}$ and $46.71 \mu \mathrm{m}$, respectively. Interestingly, if the reaction temperature maintained at $60{ }^{\circ} \mathrm{C}$, the reaction time was only $10 \mathrm{~min}$ but the obtained $\mathrm{Cu}$ NWs had an average diameter of $85.14 \mathrm{~nm}$ and an average length of $33.39 \mu \mathrm{m}$. It has been shown in previous studies that heat is required to accelerate the formation of nanowires [16]. However, excessive heat has been shown to be the cause of formation of relatively short and thick $\mathrm{Cu}$ NWs [14]. Therefore, after the reaction mixture was heated at $60{ }^{\circ} \mathrm{C}$ for only a short time ( $3 \mathrm{~min}$ ), it was cooled to RT with the expectation of obtaining longer and thinner $\mathrm{Cu}$ NWs. And the data produced in this study showed a notable increase in both the length and diameter of the $\mathrm{Cu}$ NWs when the reaction mixture was maintained at RT instead of $60^{\circ} \mathrm{C}$. Furthermore, when the mixture was cooled to room temperature, the total mass of the synthesized $\mathrm{Cu}$ NWs remarkably raised from 17.73 to $80.16 \%$. This leads to the presumption that RT conditions enhance both the aspect ratio of CuNWs and the productivity. While other reported EDA-mediated synthesis methods require reaction temperature above $60{ }^{\circ} \mathrm{C}$ for longer than $30 \mathrm{~min}$ to attain the highest yield at $60 \%$, this simple discovery will help in significantly reducing the cost of $\mathrm{Cu} N W s$ synthesis.

In addition, a sample of as-synthesized Cu NWs were prepared for X-ray diffraction analysis. X-ray diffraction (XRD) of $\mathrm{Cu}$ NWs sample was measured in the range of $2 \theta=20^{\circ}-80^{\circ}$ by step scanning on the Rigaku D/MAX2500 diffractometer (Rigaku Co., JAPAN). As shown in Fig. 2, all recorded peaks in the XRD pattern match to the face centered cubic phase of copper and no impurities can be observed.

\section{Characteristic of copper nanowires electrodes}

To fabricate various transparent conducting electrodes of $\mathrm{Cu}$ NWs, 10 coating solutions with different concentrations of $\mathrm{Cu}$ NWs were prepared and then were coated onto glass substrates with a Meyer rod, as shown in Fig. 3. The optical transmittance of the electrode was measured using PowerWave HT Microplate Spectrophotometer of BioTek and the sheet resistance of electrode was measured using "four-point probe" method. The relationship between transmittance and sheet resistance of $\mathrm{Cu}$ NWs was established in Fig. 4. The transmittance and sheet resistance increase dramatically with the decrease of $\mathrm{Cu}$ NWs density. The as-synthesized $\mathrm{Cu}$ NWs exhibited excellent performance with high transmittance and low resistance. For instance, the $\mathrm{Cu}$ NWs films displayed sheet resistances of $15,23.5,27.5 \Omega / \mathrm{sq}$ at transmittance of $72,81,82.5 \%$ at $\lambda=550 \mathrm{~nm}$, respectively. Compared with the $\mathrm{Cu}$ NWs electrodes which were fabricated with the same procedure by Stewart et al. [15], our $\mathrm{Cu}$ NWs electrode exhibited equivalent performance. Moreover, the performance of our films is similar or slightly better in comparison with other 
Fig. 1 a The reddish cake of $\mathrm{Cu}$ NWs, b video scope image of $\mathrm{Cu}$ NWs (scale $28.7 \mu \mathrm{m}$ ), c SEM image of $\mathrm{Cu}$ NWs

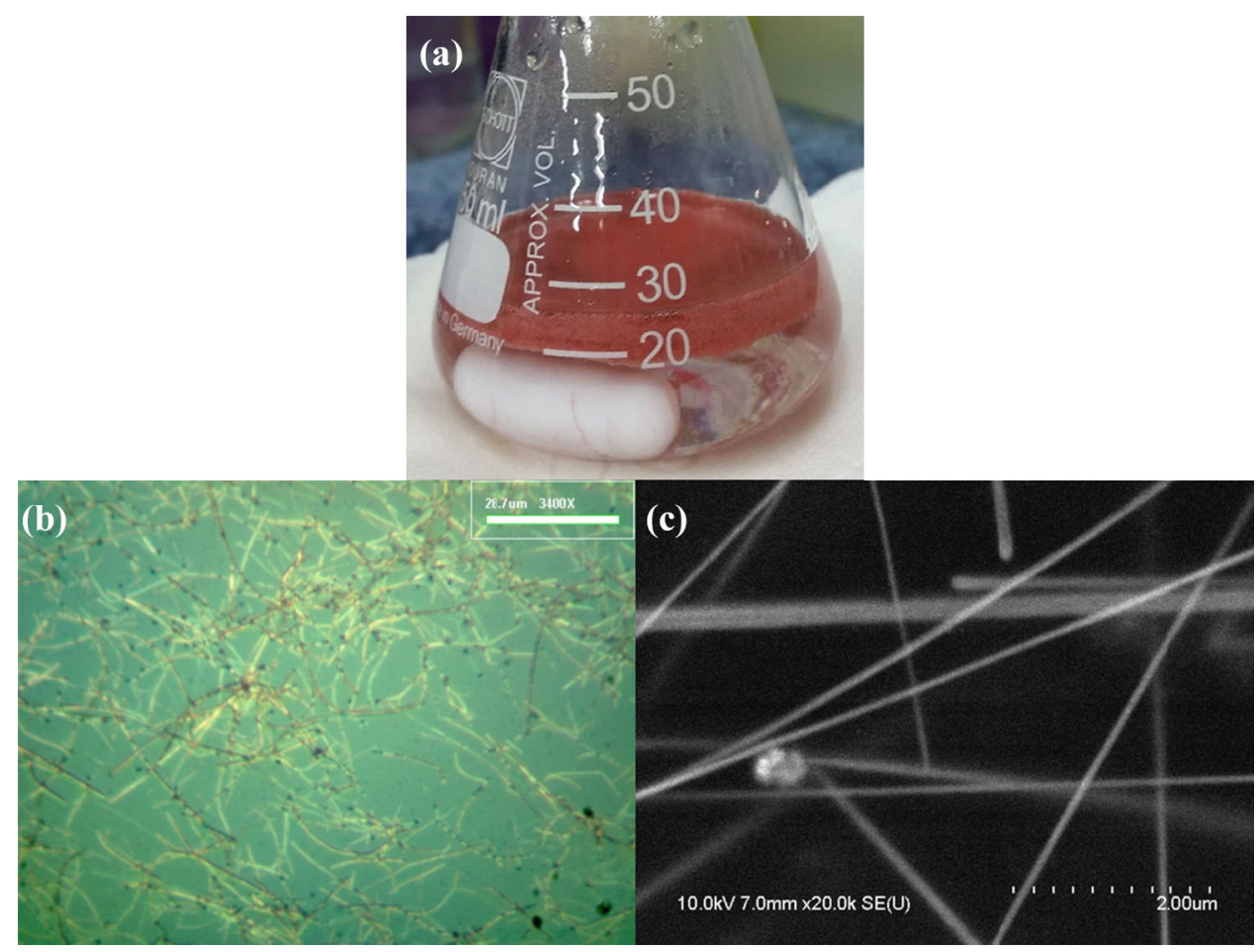

$\mathrm{Cu}$ NWs films which also went through the acetic acidtreatment step $[17,18]$.

\section{Conclusions}

In this paper, an EDA-mediated synthesis has been developed to fabricate high aspect ratio $\mathrm{Cu}$ NWs (L/D 650) within a very short time, at low cost. The yield of the $\mathrm{Cu}$ NWs produced by this method was increased outstandingly to $80.2 \%$. Furthermore, we discovered that $\mathrm{KOH}$ could speed up the formation of $\mathrm{Cu}$ NWs to reduce synthesis time to $15 \mathrm{~min}$; cooling the reaction to room temperature has a marked effect on the aspect ratio of the nanowires

Fig. 2 XRD result of as-synthesized copper nanowires

Fig. 3 Pictures of 10 samples with different concentration of CuNWs (from left to right: the concentration descends)

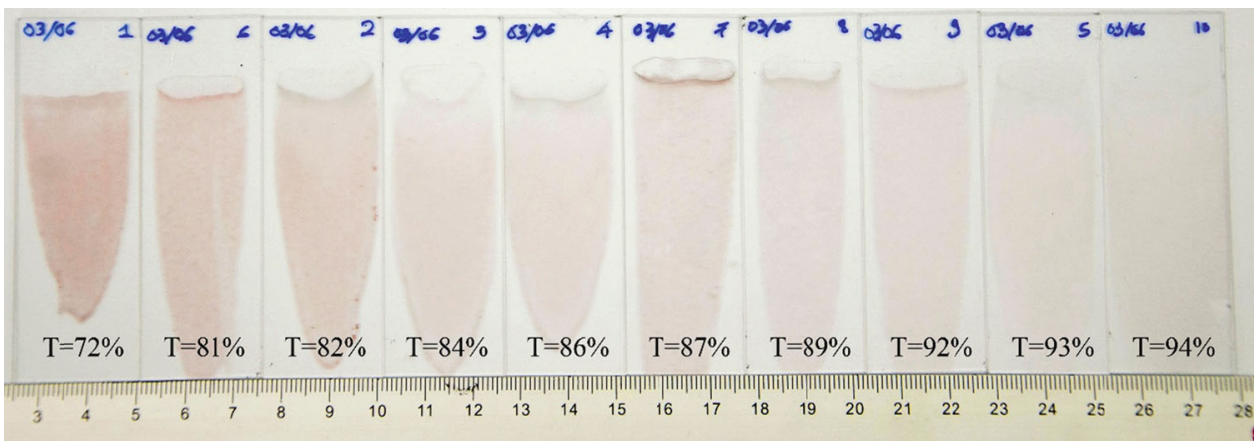




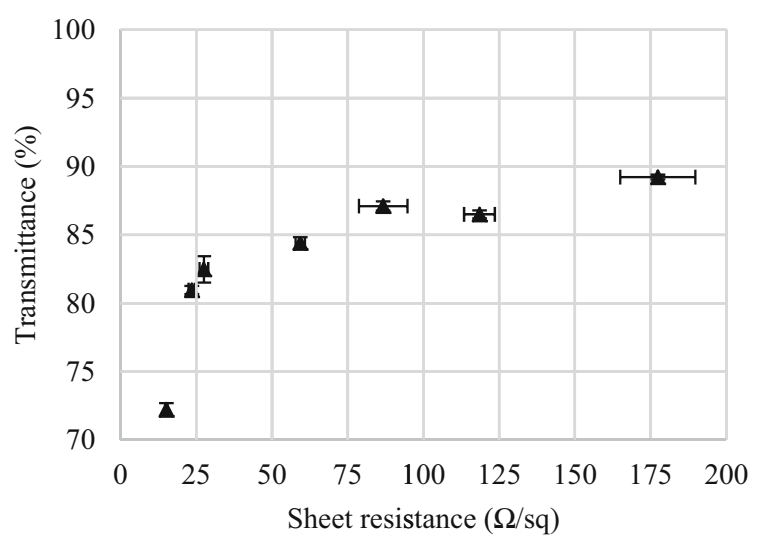

Fig. 4 Plot of transmittance $(\lambda=550 \mathrm{~nm})$ vs. sheet resistance for films of $\mathrm{Cu}$ NWs

produced. In addition, the as-synthesized $\mathrm{Cu}$ NWs were used for the fabrication of transparent electrodes and these electrodes exhibited low sheet resistance and high transmittance.

Acknowledgements This research was supported by Basic Science Research Program through the National Research Foundation of Korea (NRF) funded by the Ministry of Education, Science and Technology (2014R1A1A2053778).

Open Access This article is distributed under the terms of the Creative Commons Attribution 4.0 International License (http://crea tivecommons.org/licenses/by/4.0/), which permits unrestricted use, distribution, and reproduction in any medium, provided you give appropriate credit to the original author(s) and the source, provide a link to the Creative Commons license, and indicate if changes were made.

\section{References}

1. Hecht, D.S., Hu, L., Irvin, G.: Emerging transparent electrodes based on thin films of carbon nanotubes, graphene, and metallic nanostructures. Adv. Mater. 23, 1482-1513 (2011)

2. U.S. Geological Survey.: Mineral Commodity Summaries 2015 Mineral Commodity Summaries 2015. US Geol. Surv. 196 (2015). https://minerals.usgs.gov/minerals/pubs/mcs/2015/ mcs2015.pdf

3. Hu, L., Wu, H., Cui, Y.: Metal nanogrids, nanowires, and nanofibers for transparent electrodes. MRS Bull. 36, 760-765 (2011)
4. Ye, S., Rathmell, A.R., Chen, Z., Stewart, I.E., Wiley, B.J.: Metal nanowire networks: the next generation of transparent conductors. Adv. Mater. 26, 6670-6687 (2014)

5. Serway, R.A., Jewett, J.W.: Physics for scientists and engineers with modern physics. Brooks/Cole CENAGE Learning, Boston (2014)

6. Guo, H., Lin, N., Chen, Y., Wang, Z., Xie, Q., Zheng, T., Gao, N., Li, S., Kang, J., Cai, D., Peng, D.-L.: Copper nanowires as fully transparent conductive electrodes. Sci. Rep. 3, 2323 (2013)

7. Shi, Y., Li, H., Chen, L., Huang, X.: Obtaining ultra-long copper nanowires via a hydrothermal process. Sci. Technol. Adv. Mater. 6, 761-765 (2005)

8. Li, S., Chen, Y., Huang, L., Pan, D.: Large-scale synthesis of well-dispersed copper nanowires in an electric pressure cooker and their application in transparent and conductive networks. Inorg. Chem. 53, 4440-4444 (2014)

9. Mayousse, C., Celle, C., Carella, A., Simonato, J.: Synthesis and purification of long copper nanowires. Application to high performance flexible transparent electrodes with and without PEDOT:PSS. Nano Res. 7, 315-324 (2014)

10. Ye, S., Rathmell, A.R., Stewart, I.E., Ha, Y.-C., Wilson, A.R., Chen, Z., Wiley, B.J.: A rapid synthesis of high aspect ratio copper nanowires for high-performance transparent conducting films. Chem. Commun. 50, 2562-2564 (2014)

11. Ye, S., Rathmell, A.R., Ha, Y.-C.C., Wilson, A.R., Wiley, B.J.: The role of cuprous oxide seeds in the one-pot and seeded syntheses of copper nanowires. Small 10, 1771-1778 (2014)

12. Wiley, B., Rathmell, A.: Compositions and methods for growing copper nanowires (2013). https://www.google.ch/patents/ WO2011071885A2?cl=en. Accessed 1 Sep 2015

13. Chang, Y., Lye, M.L., Zeng, H.C.: Large-scale synthesis of highquality ultralong copper nanowires. Langmuir 21, 3746-3748 (2005)

14. Rathmell, A.R., Wiley, B.J.: The synthesis and coating of long, thin copper nanowires to make flexible, transparent conducting films on plastic substrates. Adv. Mater. 23, 4798-4803 (2011)

15. Stewart, I.E., Rathmell, A.R., Yan, L., Ye, S., Flowers, P.F., You, W., Wiley, B.J.: Solution-processed copper-nickel nanowire anodes for organic solar cells. Nanoscale. 6, 5980-5988 (2014)

16. Filipič, G., Cvelbar, U.: Copper oxide nanowires: a review of growth. Nanotechnology. 23, 194001 (2012)

17. Chen, J., Zhou, W., Chen, J., Fan, Y., Zhang, Z., Huang, Z., Feng, X., Mi, B., Ma, Y., Huang, W.: Solution-processed copper nanowire flexible transparent electrodes with PEDOT:PSS as binder, protector and oxide-layer scavenger for polymer solar cells. Nano Res. 8, 1017-1025 (2015)

18. Wang, R., Ruan, H.: Synthesis of copper nanowires and its application to flexible transparent electrode. J. Alloys Compd. 656, 936-943 (2015) 